\title{
Non-immersive Virtual Reality for Fine Motor Rehabilitation of Functional Activities in Individuals with Chronic Stroke: A Review
}

\author{
Stephanie LeBlanc ${ }^{1 *}$, Kate Paquin², Kelly Carr² and Sean Horton² \\ ${ }^{1}$ School of Rehabilitation Sciences, McMaster University, Hamilton, ON, Canada \\ ${ }^{2}$ Department of Kinesiology, University of Windsor, Windsor, ON, Canada
}

\begin{abstract}
More than two thirds of the individuals who have strokes are over the age of 65 . Therefore, as the global population continues to age, the risk of stroke is expected to increase substantially. Virtual reality (VR) is an emerging therapy that holds promise for the rehabilitation of patients with chronic stroke conditions. VR is an interactive, computer-based simulation of real life tasks, occurring in real time. The aim of this review was to explore whether non-immersive VR could be used to effectively improve fine motor function of the affected upper extremity in patients with chronic stroke. Ten studies examining non-immersive VR for the purpose of chronic stroke rehabilitation were included for review. Studies utilized a variety of VR-based interventions, reporting trends toward improvement on nearly all outcome measures. Results were examined at the levels of "body structure and function" and "activity" according to the International Classification of Functioning. Across the studies, significant improvements were reported for the Jebsen Test of Hand Function, the Box and Block Test, participants' finger fractionation, finger tracking measures, and time from peak hand velocity to movement of an object. However, considerable variability in participants' recovery rates of fine motor function across the studies suggests that the results should be interpreted with caution. More research using randomized controlled trial designs will clarify evidence surrounding the amount of improvement that can be experienced with non-immersive VR-based interventions. This review provides justification for continued investigation within the field of motor skill recovery in patients with chronic stroke.
\end{abstract}

Keywords: Virtual reality; Chronic stroke; Aging; Rehabilitation; Neuroplasticity

\begin{abstract}
Abbreviations: BBT: Box and Block Test; JTHF: Jebsen Test of Hand Function; PMP: Proximal-metacarpal-phalanx; RCT: Randomized Controlled Trial; ROM: Range of Motion; SAILS: Structured Assessment of Independent Living Skills; UE: Upper Extremity; VE: Virtual Environment; VR: Virtual Reality; ADL: Activities of Daily Living; SORT: Strength of Recommendation Taxonomy; ICF: International Classification of Functioning
\end{abstract}

\section{Introduction}

Worldwide, stroke remains the leading cause of long-term motor disability among adults [1-4]. As the population continues to age, so too, will the risk of stroke, with more than two-thirds of strokes affecting those over the age of $65[5,6]$. One of the many positive achievements of modern medicine has been the decrease in mortality rates following stroke. As such, the number of older individuals surviving stroke and in need of motor rehabilitation is predicted to see inevitable increases due to the long-term sequelae associated with stroke $[2,6,7]$. The majority of survivors of stroke will experience UE deficits, and for approximately $55-75 \%$ of these individuals, the deficits will endure well beyond the time of injury [8]. As such longstanding impairments hold the power to substantially reduce one's quality of living [9], the degree of motor and functional recovery attained is often a pivotal influence in whether a stroke is deemed debilitating [10].

\section{A Plastic Brain}

Following a stroke it is essential for the brain to undergo reorganization in order for motor recovery to occur [7]. For decades, neuroscientists suggested that the mature central nervous system was static in nature, encompassing little capacity to restructure, and by that means, repair itself $[11,12]$. However, it has since been established that not only is the human brain capable of such plastic changes, it is always changing $[11,12]$. This ongoing neural activity is appropriately termed neuroplasticity, which can be described as the brain's natural tendency to reorganize itself in response to changing internal and external demands $[1,13]$. Stroke rehabilitation has become a popular platform for neuroplasticity-related research, as motor recovery from stroke aptly demonstrates the brain's malleability and capacity for 'rewiring' post-injury [11]. It is of importance to note that this plasticity exists as a function in both healthy and damaged brains alike [14]. Furthermore, the brain's remarkable ability to promote repair following a stroke has been observed to extend years beyond the initial injury $[4,15]$.

\section{The Motor Recovery 'Plateau'}

Despite this notion of an incessantly plastic brain, the majority of recovery of general motor function has typically been observed within the first six to twelve months post-stroke $[12,16]$. The rate at which recovery occurs appears to decelerate as time passes, with recovery most rapid in the first month, slowing in subsequent months, and eventually reaching a 'plateau' $[15,16]$; that is, a point in time during the course of rehabilitation in which a patient no longer exhibits signs of improvement in response to therapeutic intervention [15]. This observable plateau holds serious implications for stroke survivors, as this perceived cessation of progress often provides the grounds for discharge from rehabilitative programs $[17,18]$. As such, reservations regarding the cost-effectiveness are warranted, since the apparent widespread presence of a motor recovery plateau suggests a limit to

*Corresponding author: Stephanie LeBlanc, School of Rehabilitation Sciences, McMaster University, Canada, E-mail: leblansm@mcmaster.ca

Received March 14, 2013; Accepted March 16, 2013; Published June 18, 2013

Citation: LeBlanc S, Paquin K, Carr K, Horton S (2013) Non-immersive Virtual Reality for Fine Motor Rehabilitation of Functional Activities in Individuals with Chronic Stroke: A Review. Aging Sci 1: 105. doi:10.4172/2329-8847.1000105

Copyright: ( 2013 LeBlanc S, et al. This is an open-access article distributed under the terms of the Creative Commons Attribution License, which permits unrestricted use, distribution, and reproduction in any medium, provided the original author and source are credited. 
late functional recovery of survivors of stroke [19,20]. Accordingly, as stroke recovery nears the more stable, chronic stages (greater than twelve months post-stroke), doubt pertaining to the effectiveness of a motor-rehabilitation intervention rises, and patients are often denied any further treatment [19-21]. Nevertheless, this proposed motor recovery plateau has not gone without opposition. Rather than explain stroke-related plateaus as a diminished capacity to manifest any further motor gains, Page, Gater and Bach-y-Rita (2004) have likened the plateau to the neuromuscular adaptation that occurs after exercise in healthy adults. Despite operating on similar principles, the common response to such adaptations differs greatly. When a healthy adult experiences neuromuscular adaptation to exercise, the routine is subsequently varied or intensified to facilitate positive change; not terminated [18]. It has been argued that individuals with strokes experience neuromuscular adaptations to rehabilitative exercise, however, in contrast to their healthy counterparts this adjustment to treatment commonly results in the discontinuation of therapy [18]. In addition to disagreements surrounding the nature of the plateau, some question its susceptibility to external influences $[17,18]$. Demain et al. [17] suggest that the plateau is inherently complex, and expose the ambiguity surrounding its current conceptualizations. There is reason to believe that patient-related factors, therapist values, services provided, and the dynamic patient-therapist relationship all play a part in a patient's respective recovery [17]. Both Page et al. [17] and Demain et al. [18] note that a therapist's acceptance of such a plateau may in fact limit patients' expectations for recovery, thereby inhibiting success by way of a self-fulfilled prophecy. Though the mechanics underlying the plateau are not entirely understood, promise that the motor recovery plateau can be overcome is held, to some extent, in the emergence of successful cases of chronic-stroke recovery [15,20,22-31].

\section{Rehabilitation for 'Chronic' Stroke}

The window of time for the effective application of restorative therapies is not entirely clear, and remains highly variable between patients [15]. Although early rehabilitation has been deemed more effective, motor recovery in the chronic stages of stroke has been observed, and can be attributed, in part, to physical rehabilitation [15,17,22-31]. A meta-analysis conducted by Ferrarello et al. [20] concluded that in comparison to no treatment or a placebo, motor rehabilitation applied to patients with chronic strokes improved both motor and functional outcomes of recovery. Still, due to the scarcity of high-quality, conclusive evidence of the effectiveness of late rehabilitation, it is uncommon for patients having chronic stroke to be offered physical therapies [20,32]. Evidently, further research surrounding the cost-effectiveness, practicality, and best practice of late rehabilitation is required before any definitive change can be made $[9,20]$.

\section{Virtual Reality}

With a greater understanding of the nature of brain plasticity, stroke rehabilitation continues to gravitate toward therapeutic approaches that capitalize on these insights, in an effort to address the limitations of conventional rehabilitation practices, and to optimize functional outcomes [33,34]. Among these approaches is VR, a computerbased technology that allows users to interact with a simulated environment and receive continuous, immediate feedback related to performance [34-36]. Since motor recovery following stroke has been found to be experience-dependent, it is vital to employ rehabilitative interventions that facilitate quality experiences, which serve to bolster neuroplastic change $[9,33]$. Kleim and Jones [33] highlighted several principles related to experience-dependent neuroplasticity that can be incorporated into rehabilitation of the damaged brain, including intensity, repetition, specificity, and salience. Intensity refers to the number of hours of consecutive therapy that a patient receives, whereas repetition refers to the number of times a particular learned behavior is practiced [33]. Table 1 (Included as supplementary data) outlines the studies' protocols as well as the total number of hours of therapy received by each participant. Specificity is used in reference to the training of specific purposeful and skilled tasks, with the intent of learning or relearning that task [33]. Last, salience relates to a patient's engagement in therapy, achieved through sufficient motivation and attention, which adds greater level of importance to the task [33]. VR lends itself well to the application of such principles, given its capacity to encompass task-specific training, appropriate intensities and repetition, and salient experiences [33,35-39]. Furthermore, VR systems can be tailored to the individual needs of a patient, to include meaningful, challenging, and progressive exercises that can be carried out in a variety of settings. This flexibility supports high-intensity, repetitious training that patients find motivating, engaging, and enjoyable [22,30,33,40]. VR-based therapy can be classified on a continuum from fully immersive to nonimmersive, dependent on the degree to which the user is perceivably immersed in the VE $[34,41]$. Immersive VR allows the user to feel that they are situated within the VE presented to them; a concept termed "presence" [36]. This presence can be achieved and reinforced through the use of a variety of equipment both worn by, and in front of the user (e.g., cave systems, large screen projections, head mounted displays, and specially designed stereoscopic glasses) $[27,36]$. In contrast, nonimmersive VR can be likened to looking through a window at a scene, and often involves the use of smaller-scale, 2-dimensional screens (e.g., computer or television screens), with or without the use of interface devices (e.g., Cyber glove, joy stick, or computer mouse) [27,36,41,42]. Also considered non-immersive VR are commercially available computer and video gaming systems [34]. These systems have been adopted by clinicians as accessible and relatively low-cost alternatives to expensive therapeutic technologies, though not specifically designed for rehabilitative purposes [34].

\section{The Benefits and Challenges of Virtual Rehabilitation}

Promising evidence exists for the use of non-immersive VR as a supplement to conventional rehabilitation practices for persons with stroke [41,43-46]. As with any modality for stroke rehabilitation, the implementation of VR is accompanied by both benefits and challenges (Table 2). This is by no means an exhaustive, nor an indisputable listing; however the abundance of perceived benefits warrants further exploration of VR for therapeutic purposes. The identified challenges are justified given the relative novelty of virtual rehabilitation, and may partially be founded in unfamiliarity, among both therapists and clients, regarding both the technology and its potential value within therapeutic settings. As such, some of these challenges will perhaps be resolved through continued exposure and enhanced awareness [4750]. Nonetheless, the numerous challenges will need to be addressed through additional research in order for a widespread acceptance of VR for rehabilitation to materialize.

\section{Purpose of Review}

Associated with our aging population is an increasing demand for stroke-related motor rehabilitation [5,6]. As UE deficits have been found to endure for years beyond injury in some patients, the need for effective late-rehabilitation practices is of vital importance [8]. Stroke-induced hemiparesis affects fine motor control, which can dramatically impair 
Citation: LeBlanc S, Paquin K, Carr K, Horton S (2013) Non-immersive Virtual Reality for Fine Motor Rehabilitation of Functional Activities in Individuals with Chronic Stroke: A Review. Aging Sci 1: 105. doi:10.4172/2329-8847.1000105

Page 3 of 8

\section{Benefits}

Provision of a non-threatening practice environment, which may prove dangerous otherwise (e.g., preparing a hot beverage or operating a motor vehicle) [47]

Simulation of objects and events that closely resemble those in real-world settings [43]

Flexibility that allows for highly individualized learning environments [48]

Ability to accommodate for a wide spectrum of cognitive and physical impairment $[29,40,47]$

Low cost associated with commercially available VR systems $[47,49]$

Potential to increase patient enjoyment, motivation, and thereby adherence $[29,40,47,49]$

The potential for telerehabilitation in a variety of patient environments, absent of direct therapist supervision $[40,49,50]$

Adjustable task-related variables according to patient's abilities (e.g., increasing the speed of tasks based on a patient's progress) [29]

Digital measurement and tracking of patients' progress [47]

Constraints to encourage error-free learning (e.g., a virtual piano only emitting sound when the correct key has been pressed) [47]

Increased independent practice time, and task repetition [29,40,47,49]

Same VR hardware can be used for various different exercises, as well as for various different patients $[40,47]$

Offers real-time visual and potentially force feedback to user $[29,47]$

\section{Challenges}

Therapists' resistance to technology (e.g., for fear of being replaced, or lack of conviction in its effectiveness) $[40,47]$

Lack of clinical acceptance due to the paucity of evidence surrounding factors such as cost effectiveness or feasibility $[40,47]$

Many virtual interfaces have not been designed for medical purposes, raising issues of patient safety (e.g., adequate sterilization for repeated use may be problematic) $[36,40]$

Commercial equipment that is unable to adequately accommodate all physical impairments [36,40]

Unaffordability of VR software and associated hardware for individuals and organizations that lack subsidy $[36,40]$

Patients' negative perception of VR [36]

Lack of patient compliance in the absence of direct therapist interaction [40]

Table 2: Overview of the perceived benefits and challenges of non-immersive VR for stroke rehabilitation extracted from the literature.

a person's ability to live independently, perform ADL and participate in leisure activities and productive work [51,52]. Non-immersive VR is a rehabilitative approach that has gained support in recent years, and shows promise for its ability to promote motor restoration through the application of neuroplastic principles [36]. Therefore, the purpose of this review is to explore the effectiveness and user perspectives of nonimmersive VR for the rehabilitation of fine motor skills of the UE in patients with chronic stroke.

\section{Methods}

\section{Identification of relevant studies}

The literature was reviewed by three researchers to identify published studies including RCT case studies, and pre-test/posttest study designs that focused on the use of non-immersive VR in fine motor UE rehabilitation of patients with chronic stroke (more than 12 months post-stroke). The following databases were searched: OVID-MEDLINE, EMBASE, Scopus, CINAHL, Pro Quest Science and Technology, and Google Scholar. These databases were searched using the following key terms:"virtual reality", "virtual environment", "commercial gaming", and "non-immersive" in combination with "stroke", "rehabilitation", "treatment", "fine motor" and "chronic" or "late-stroke". Additionally, the reference lists of each retrieved article were reviewed in order to identify other pertinent articles.

\section{Eligibility criteria}

Inclusion criteria were (1) English-language articles that examined the effectiveness of (2) non-immersive VR for (3) fine motor rehabilitation of the UE in (4) individuals having chronic stroke, and (5) studies incorporating a combination of both VR-based and conventional exercises (i.e., augmented VR). Exclusion criteria were (1) conference proceedings and abstracts, (2) studies on individuals with acute or subacute stroke conditions at the onset of the study (less than 12 months post-stroke), (3) studies utilizing specially designed assistive technology, (4) studies focusing on gross motor rehabilitation (e.g., gait or balance-related activities), (5) studies using fully immersive
(VR) technology (e.g., large screen projections, cave systems, headmounted displays, force-plate technology, and assistive robotics). Last, (6) wrist measurements alone did not suffice as grounds for inclusion when involved in gross motor movements, such as reaching (e.g., wrist displacement), as fine motor will refer only to those manual movements predominantly produced by the smaller muscles or muscle groups of the UEs.

\section{Quality appraisal}

The methodological quality of the included studies was assessed according to the SORT [53]. Studies were not excluded on the basis of the SORT evaluation; rather, this information was used to substantiate recommendations according to the quality, quantity and consistency of the reviewed studies. According to the SORT, the quality of individual studies was rated 1,2 , or 3 , while the strength of a recommendation based on the overall body of evidence was graded as A, B, or C [53]. A level-1 indicated good quality, patient-oriented evidence, a level-2 indicated. limited-quality, patient-oriented evidence, and a level-3 indicated non-patient-oriented evidence or other evidence [53]. In terms of the strength of recommendation, a grade-A indicated a recommendation based on consistent and good-quality, patientoriented evidence, a grade- $\mathrm{B}$ indicated a recommendation based on inconsistent or limited-quality patient-oriented evidence, and a grade- $\mathrm{C}$ indicated a recommendation based on consensus, usual practice, opinion, disease-oriented evidence, or case series for studies of diagnosis, treatment, prevention, or screening [53]. According to these criteria, the selected studies were rated as level-1 $(n=1)$, level-2 $(n=4)$ and level-3 $(n=5)$, with an overall recommendation strength grade- $B$ (recommendation based on limited-quality patient-oriented evidence). Please refer to Table 1 (Included as supplementary data) for specific SORT levels.

\section{Sample study characteristics}

Nine research articles provided the ten selected studies under review ([26] published two research studies within a single article). The majority of the ten selected studies were case studies $(n=5)$, followed by 
studies using a pre-test/post-test design $(\mathrm{n}=4)$, and one study employed a RCT design. Most studies had participants engage solely in VRbased rehabilitation strategies $(\mathrm{n}=8)$ with three studies augmenting VR rehabilitation strategies with traditional exercises to improve fine motor skills. With respect to all of the articles reviewed, the participants' $(n=60)$ ages ranged from 42 to 85 years, with participants between 1 and 8 years post-stroke ( Table 1 (Included as supplementary data)).

\section{Outcome measures}

Within the ten selected studies, a variety of methods were used to measure fine motor skills of the affected hand post-stroke (Table 1 (Included as supplementary data)). At the "body structure and function" level of the ICF [54], outcome measures were finger and hand movement parameters, including measures of finger and thumb speed and strength, finger and thumb ROM, finger fractionation, peak hand velocity, kinematic analysis of prehension movements, and finger tracking accuracy. Similarly, at the "activity" level of the ICF [54], outcomes included the JTHF; $(n=8)$, the BBT $(n=1)$, and the SAILS $(\mathrm{n}=1)$. Eight studies involved measurement at both "body structure and function" and "activity" levels to assess fine motor ability, while the remaining two studies used only measures of "body structure and function" or "activity" [54]. The JTHF was developed as a short, objective evaluation of basic hand functions relative to ADL, and consists of seven subsets that provide a broad sampling of functional tasks [55]. The test items include a range of fine motor, weighted and non-weighted manual activities, including writing, turning index cards, picking up small common objects, simulated feeding, stacking checkers, picking up large light objects, and picking up large heavy objects [55]. The BBT is a quick and simple measure of unilateral gross manual dexterity [56]. It requires participants to grasp and move as many blocks within 60 seconds from one side of a divided box with the tip of the index finger and tip of the thumb of the paretic hand and release the block on the opposite side of the box [24]. The SAILS is an assessment of functional abilities associated with ADL [57]. It directly assesses 10 areas of everyday functioning: fine motor skills, gross motor skills, dressing, eating, expressive language, receptive language, time and orientation, money-related skills, instrumental activities, and social interaction [57].

\section{Results}

The results from ten research studies examining VR-based rehabilitation for fine motor skills during the chronic phase of stroke have been reviewed. According to a SORT assessment the quality of the individual studies ranged from level-1 through level-3, with an overall strength of recommendation of grade-B. The study type, SORT level, specific exercises performed, intervention protocol, total hours of VR exercise, and the outcome measures for each study are included in Table 1 (Included as supplementary data). To avoid redundancy, we have identified all outcome measures that were used on one occasion. Please refer to Table 1 (Included as supplementary data) for specific results. Outcome measures utilized in two or more studies are compared and summarized below.

\section{Body structure and function level}

Finger and thumb ROM: Results of seven studies unanimously indicated a trend toward improvement in finger and thumb ROM following VR-based rehabilitation [22,24,26,28-30]. While improvements were found in each of these studies, only two reported findings that reached a level of statistical significance [24,30]. Carey et al. [24] reported that finger and thumb ROM significantly increased in participants provided with a pathway to follow on the virtual interface (track group), while participants without this pathway (move group), experienced no change. As only the track group was trained to increase their ROM using tracking protocols with amplitudes at $125 \%$ of their range, these findings were expected. Merians et al. [30] also reported a significant improvement from pre-therapy to posttherapy, with performance after a one-week retention period remaining significantly better than pre-therapy performance. It is worth noting that individually reported data contained in four of the seven articles measuring finger and thumb ROM revealed a wide range of change in thumb (-40\% to $148 \%)$ and finger ROM (-9 to $27 \%)$ suggesting little consensus regarding expected improvement in finger and thumb ROM through VR rehabilitation late-stroke $[26,28,30]$.

Finger and thumb speed: Six studies assessed finger and thumb speed [22,26,28-30]. While five of these reported improvements following VR rehabilitation, only one study reported findings at a level of statistical significance, as measured by a CyberGlove [30]. Furthermore, when assessed following a one-week retention period it was found that finger and thumb speed were significantly better than pre-therapy levels [30]. Despite overall positive outcomes, individualized rates of recovery (presented as a percentage) demonstrated considerable variability, with thumb speed change ranging from $-7 \%$ to $80 \%$, and finger speed change from $-1 \%$ to $78 \%[26,28,29]$.

Finger fractionation: Six studies reported improvements in finger fractionation (i.e., the assessment of participants' isolated finger control) following VR therapy [22,26,28-30], however only two of these studies reported significant improvements [22,30]. Merians et al. [30] reported that these improvements were maintained above pre-therapy levels after one week without participating in VR rehabilitation. Similar to the above impairment measures, there was considerable variability regarding the improvement in finger fractionation of chronic stroke patients, with changes ranging from $-22 \%$ to $118 \%$, with an average increase of $50 \%$ [22,26,28,29].

Ability of fingers and thumb to do mechanical work: Mechanical work was estimated as the force exerted by the thumb or fingers in relation to their displacement [30]. One of five studies examined the statistical significance of changes in mechanical work done by the fingers and thumb, and reported that there was no significant increase in this measure of fine motor skills [30]. However, through the use of percentage measures, the remaining four studies suggested a $29 \%$ average increase in the ability of the fingers and thumb to do mechanical work. This increase should be interpreted with caution as the results varied greatly, with changes ranging from $-18 \%$ to $102 \%[26,28,29]$.

Peak hand velocity: Two studies examined the time elapsed from peak hand velocity to the moment an object is lifted off a table [22,30]. Adamovich et al. [22] reported no change in time to peak velocity following VR therapy, however, this finding was to be expected as participants' elbow and shoulder were not trained during therapy. In contrast, time from peak velocity to the moment the object was lifted from the table did decrease significantly; performed $22 \%$ faster on average following intervention [22]. This finding suggested an increase in the participants' ability to appropriately match their finger positions to the shape of the object [22,30]. Similarly, Merians et al. [30] found that despite a lack of change in peak hand velocity, participants' time from peak velocity to the moment the object was lifted from the table significantly decreased. On average, participants performed this task 19\% faster after the intervention, again illustrating a transfer of improvement to a real-world task [30]. 


\section{Activity level}

Jebsen Test of Hand Function: Eight studies used the JTHF as an outcome measure, primarily to assess the transferability of improvements to functional tasks [22,24-26,28-30]. Overall, the studies suggest that VR rehabilitation during the chronic phase of stroke can significantly improve performance on the JTHF [22,24,26,30]. Though the remaining studies that used the JTHF as a measure of fine motor skills did not provide significance values, each provided results indicative of improvement following VR therapy [25,26,28,29].

\section{Discussion}

Stroke is the leading cause of long-term disability among adults [14]. In order for motor recovery to occur following an injury such as a stroke, neural reorganization is imperative [7]. Neuroplasticity, a term used to describe this reorganization of the brain, has sparked substantial research surrounding motor recovery post-stroke [1,13]. However, there is a limited number of quality studies focused specifically on the use of VR interventions for fine motor rehabilitation in chronic stroke patients. Since VR is a novel rehabilitative approach within this population, it is important to review the preliminary evidence to allow for meaningful and timely progression. Therefore, the purpose of this review was to examine the effectiveness of non-immersive VR-focused rehabilitation for improving UE fine motor skills in chronic stroke patients. As a whole, the articles reviewed suggest that VR rehabilitation may yield positive changes in individuals' motor recovery in the years following their stroke. Within the ten studies, a variety of methods were used to measure fine motor skills of the affected UE post-stroke, at both the "body structure and function" and "activity" levels of the ICF [54]. At the level of "body structure and function," several measures indicated improvements, with the exception of measures of finger strength and finger extension [22-26,28-30,58]. Among these measures, significant improvements were found in participants' finger fractionation $[22,30]$, finger tracking [24], and time from peak hand velocity to the moment an object was lifted from a table $[22,30]$. However, these results should be interpreted with caution given the considerable variability between participants' degree of change. At the level of "activity" [54], the JTHF, BBT, and SAILS were used as measures. These measures were primarily used to determine transferability of gains from VR therapy to real-world tasks. The JTHF was utilized by eight of the reviewed studies; of which five reported significant improvements [22,24,26,30]. Furthermore, significant improvement in participants' performance on the BBT was reported, however no change was reported in the participants' SAILS scores following VR intervention [24,40]. Included within this review, only a single study utilized either of these measures (i.e., the BBT or SAILS), making comparison between studies impossible. Further research utilizing these particular outcome measures is required in order for the development of any definitive conclusions.

\section{Experience-induced neuroplasticity}

It is feasible that the positive findings throughout the reviewed studies were at least partially a result of the facilitation of cortical reorganization by VR intervention. Each of the studies demonstrated the application of various neuroplasticity-bolstering principles, including task-specific training, high intensity and repetition, salience, and novelty [33]. As previously mentioned, the intensity of each intervention was operationalized as the total number of hours of consecutive therapy a participant received. Intensities (Table 1 (Included as supplementary data)), ranged from a minimum of 15 minutes to a maximum of 5 hours of total therapy per day. When considered with the duration of each study, the total number of hours of therapy received by each participant ranged from 2.5 hours to 45 hours. Despite similar findings among studies, there remains a lack of definitive conclusions about appropriate intensities due to the variability of these components in the study protocols. For example, significant improvements on the JTHF were reported by studies with a wide range of intensities and durations $[22,24,26,30]$. This would suggest that mechanisms beyond intervention intensity were responsible for these gains in function, or that just 15 minutes of intensive therapy each day might be sufficient in producing such gains. However, it is of importance to note that despite its short duration and intensity (minimum 2.5 hours, and minimum 15 minutes per day, respectively) this intervention employed a high degree of repetition [24]. That is, each participant was required to perform 180 trials (each lasting between 5 and 15 seconds) each day, for 10 days [24]. Therefore, this high degree of task-specific repetition may have been sufficient for facilitating neuroplastic changes.

Another important mechanism of neuroplasticity, perhaps compensating for lower intensities and short durations, was likely the salience facilitated by each of the VR interventions [33]. Though not explicitly stated consistently, the novelty and 'play' component associated with each of the VR interventions is believed to have fostered greater participant enjoyment, motivation, and thereby engagement as compared to conventional rehabilitation practices [20,30,33]. It is possible that participants' attention was heightened and that skills were performed with greater purpose and effort. Therefore, the novelty, interactive nature, and game-like characteristics may have fulfilled the requirements of salience needed for the learning of tasks, resulting in greater neuroplastic change and overall functional gain [33]. Within the studies reviewed, the notion of VR-augmented therapy was explored $[26,28,29]$. The conventional exercises encompassed functional fine motor tasks, including: the placement of paper clips $[26,28,29]$, moving checkers [28,29], and arranging pegs on a pegboard $[28,29]$. Interestingly, all three augmented VR studies noted an increase in thumb ROM, whereas only one of the standalone VR studies did [26]. Beyond increased thumb ROM, the augmented VR studies yielded similar results to the standalone VR studies (e.g., improved finger fractionation, finger speed, and JTHF scores) [22,24,26,28]. This offers practical implications for therapists and researchers without the means necessary to run a conventional rehabilitation program, or for those looking to augment an existing program with VR. Researchers questioned whether the changes found in participants' performance were due to VR training, conventional training (i.e., non-VR training) that augmented the VR training, or a combination of the two [28] Merians et al. [30] opted for the elimination of non-VR tasks from the study thereby crediting any changes in functioning to VR-based interventions. In support of this objective, Merians et al. [30] found that training in an environment that was entirely VR-based elicited motor recovery changes that did in fact translate to real world functional tasks, as demonstrated by changes on the JTHF. Further research comparing outcome differences between augmented and standalone VR in chronic stroke is needed.

\section{Benefits and challenges}

Key benefits noted throughout the literature reviewed were participants' enjoyment, motivation, engagement in, and acceptance of VR therapies $[23,26,28,29,41]$. Researchers explored the positive influences of VR interventions through the monitoring of participants' attendance, mood, engagement, and willfulness to adapt to the VR training $[23,26,28,29]$. Interestingly, participants who initially expressed a negative attitude toward VR training developed a more positive attitude or 'spirit' over time [23]. Such positive attitudes 
were exemplified through maintained attendance, including punctual attendance and willingness to make up for missed appointments, throughout the intervention [23]. Following one VR intervention, participants reported feeling that continued participation in VR would lead to further gains, and wished that they had participated in VR therapy sooner [28]. Similarly, Lewis et al. [59] found that despite a lack of clinically significant gains, perceived gains were made in participants' hand function, and participants reported increased ease in terms oftheir performance of ADL.

\section{User perspectives}

The sustainment of VR-based interventions in physical rehabilitation is heavily influenced by the perspectives of its users [59]. Lewis and colleagues examined user perspectives toward a VR intervention in order to better understand which of its aspects were considered most important. Several themes emerged across user responses, including: (1) stretching oneself, (2) purpose and expectations, and (3) future improvements. Stretching oneself related to performing a novel activity and challenging oneself physically, mentally and socially [59]. In challenging participants' current ability, participants reported stretching themselves into areas that they had not previously explored. Responses pertaining to purpose and expectations of VR revealed that the users only wished to use VR in the context of rehabilitation, as opposed to using it for entertainment purposes [59]. Expectations of VR were influenced by previous experiences in rehabilitation, with users reporting that augmented VR in rehabilitation was most favorable. The users also offered ideas for future improvement surrounding the VR intervention [59]. The most common suggestions included: improvements to the scoring systems to include increased provision of performance feedback, decreasing distractions, enhancing the realism and accuracy of the VE, and the incorporation of a competitive component [59]. Ultimately, acceptance of a VR intervention relies on participants' ability to achieve control, experience success, and maintain an environment in which they are constantly challenged and thereby progressing [59]. A VR intervention that facilitates the experience of these factors holds the ability to promote enjoyment, motivation, and heightened commitment to the rehabilitation process as a whole $[41,59]$.

\section{Commercial gaming as a cost-effective alternative}

The costs associated with VR systems can make their use in rehabilitation settings impractical for patients and therapists alike [30]. A more affordable alternative to clinically designed VR is commercial gaming systems. To date, academic literature focused on the use of commercial gaming for chronic stroke rehabilitation is lacking. Furthermore, the paucity of research studies examining commercial gaming for fine motor rehabilitation in individuals with chronic stroke meant that an accurate portrayal of its effectiveness could not be ascertained. Due to its prospects as an inexpensive modality for VR therapy however, findings related to commercial gaming for rehabilitation of the UE post-stroke will be briefly explored. Commercial gaming has evolved into a user-friendly and affordable entity, in comparison to its preexisting models [40]. As such, community level rehabilitation settings with limited access to funding may now be able to afford commercially available VR systems [49]. This possibility holds substantial promise for those with chronic stroke, as it is predominantly through community-based rehabilitation programs that stroke patients receive therapy following discharge from inpatient and outpatient services [49]. This potential for application extends beyond community-based rehabilitation and may also be implemented as a means for home-based therapies [60]. Rehabilitation intervention studies utilizing commercial gaming have provided further support for its effectiveness in eliciting functional gains and trends toward improved quality of life $[51,61,62]$.

\section{Limitations and future directions}

The generalizability of the findings in this review is limited for a number of reasons. Neither stroke type, nor severity were reported or analyzed, however, both hold several practical implications for the applicability of the findings. Future studies should investigate the impact that the severity and type of stroke has on motor recovery outcomes following VR treatment in the chronic stage of stroke. Intensity and duration of the treatment protocols, as well as outcome measures among the included studies varied considerably, hampering our ability to make robust conclusions about the findings due to this heterogeneity. With respect to the inclusion of studies, only published studies were included which may have resulted in a publication bias toward more positive results. Last, the quality appraisal of the evidence according to the SORT [53], revealed the studies as being primarily level-2 or -3 , allowing for grade- $B$ recommendations based on their cumulative findings. The rating of the studies is indicative of limited-quality, patient-oriented evidence and case studies, which hinders our ability to indisputably attribute positive gains to VR-based interventions. The included studies provide a strong foundation for future research conducted in this area; however, evidence-based recommendations for practice contexts based on these findings are necessarily speculative at this time, provided that research surrounding VR for fine motor rehabilitation of individuals with chronic stroke is in its infancy.

\section{Conclusion}

It was once believed that the brain was a static and irreparable entity $[11,12]$, however, there is now growing evidence to support examination of the long-term rehabilitative effects following a stroke, thereby warranting research beyond the gains achieved solely during the acute and sub acute stages of stroke recovery $[11,12,15,20,22-26,28$ 31]. Although not yet widely established in stroke rehabilitation, VR training possesses the qualities to be a valuable rehabilitative tool for motor function in chronic stroke patients. The use of VR as a tool for patients in the late stages of stroke recovery has been explored for over a decade; however the number of studies concerned with fine motor rehabilitation remains limited. As evidence accumulates in support of this notion, patients with chronic stroke who were once believed to be incapable of further rehabilitative gains will have greater opportunity to work toward furthering their motor recovery. It is well known that the recovery of both gross and fine motor movements is important for the completion of ADL, and thereby, an increased quality of life $[51,52]$. As such, further research into the rehabilitation of fine motor control post-stroke should be conducted in order to determine just how viable and effective VR is as a therapeutic medium among those with chronic stroke.

\section{References}

1. Rossini PM, Calautti C, Pauri F, Baron JC (2003) Post-stroke plastic reorganisation in the adult brain. Lancet Neurol 2: 493-502.

2. Sacco RL, Frieden TR, Blakeman DE, Jauch EC, Mohl S (2012) What the million hearts initiative means for stroke: A presedential advisory from the American Heart Association/American Stroke Association. Stroke 43: 924-928.

3. Sharma N, Cohen LG (2010) Recovery of motor function after stroke. Dev Psychol 54: 254-262.

4. Ward NS, Cohen LG (2004) Mechanisms underlying recovery of motor function after stroke. Arch Neurol 61: 1844-1848. 
Citation: LeBlanc S, Paquin K, Carr K, Horton S (2013) Non-immersive Virtual Reality for Fine Motor Rehabilitation of Functional Activities in Individuals with Chronic Stroke: A Review. Aging Sci 1: 105. doi:10.4172/2329-8847.1000105

5. Russo CA, Andrews RM (2008) Hospital stays for stroke and other cerebrovascular diseases, 2005. Healthcare cost and utilization Project Statistical Brief \#51. Rockville, MD: Agency for healthcare research and quarterly, May 2008.

6. Saposnik G, Cote R, Phillips S, Gubitz G, Bayer N, et al. (2008) Stroke outcome in those over 80: A multicenter cohort study across Canada. Stroke 39: 23102317.

7. Teasell R, Bayona NA, Bitensky J (2005) Plasticity and reorganization of the brain post stroke. Top in Stroke Rehabil 12: 11-26.

8. Levin MF, Knaut LA, Magdalon EC, Subramanian S (2009) Virtual reality environments to enhance upper limb functional recovery in patients with hemiparesis. Stud Health Technol Inform 145: 94-108.

9. Dimyan MA, Cohen LG (2011) Neuroplasticity in the context of motor rehabilitation after stroke. Nat Rev Neurol 7: 76-85

10. Cramer SC, Sur M, Dobkin BH, O’Brien C, Sanger TD, et al. (2011) Harnessing neuroplasticity for clinical applications. Brain 134: 1591-1609.

11. Hallett M (2001) Plasticity of the human motor cortex and recovery from stroke. Brain Res Rev 36: 169-174.

12. Taub E, Uswatte G, Elbert T (2002) New treatments in neurorehabilitation founded on basic research. Nat Rev Neurosci 3: 228-236.

13. Lillard AS, Erisir A (2011) Old dogs learning new tricks: Neuroplasticity beyond the juvenile period. Dev Rev31: 207-239.

14. Hubbard IJ, Parsons MW, Neilson C, Carey LM (2009) Task-specific training Evidence for and translation to clinical practice. Occup Ther Int 16: 175-189.

15. Cramer SC (2008) Repairing the human brain after stroke: I. Mechanisms of spontaneous recovery. Ann Neurol 63: 272-287.

16. Skilbeck CE, Wade DT, Hewer RL, Wood VA (1983) Recovery after stroke. J Neurol Neurosur and Ps 46: 5-8.

17. Demain S, Wiles R, Roberts L, McPherson K (2006) Recovery plateau following stroke: Fact or fiction? Disabil Rehabil 28: 815-821.

18. Page SJ, Gater DR, Bach-y-Rita P (2004) Reconsidering the motor recovery plateau in stroke rehabilitation. Arch Phys Med and Rehabil 85: 1377-1381.

19. Ernst $E$ (1990) A review of stroke rehabilitation and physiotherapy. Stroke 21: 1081-1085.

20. Ferrarello F, Baccini M, Rinaldi LA, Cavallini MC, Mossello E, et al. (2010) Efficacy of physiotherapy interventions late after stroke: a meta- analysis. J Neurol Neurosur P 82: 1-28.

21. Byl N, Roderick J, Mohamed O, Hanny M, Kotler J, et al. (2003) Effectiveness of sensory and motor rehabilitation of the upper limb following the principles of neuroplasticity: Patients stable poststroke. Neurorehabil Neural Repair 17 176-191.

22. Adamovich SV, Merians AS, Boian R, Lewis JA, Tremaine M, et al. (2005) A virtual reality-based exercise system for hand rehabilitation post-stroke. Presence 14: 161-174

23. Burdea G, Cioi D, Martin J, Rabin B, Kale A, et al. (2011) Motor retraining in virtual reality: A feasibility study for upper-extremity rehabilitation in individuals with chronic stroke. J Phys Ther Educ 25: 20-29.

24. Carey JR, Durfee WK, Bhatt E, Nagpal A, Weinstein SA, et al. (2007) Comparison of finger tracking versus simple movement training via telerehabilitation to alter hand function and cortical reorganization after stroke. Neurorehabil Neural Repair 21: 216-232.

25. Deutsch JE, Merians AS, Burdea GC, Boian R, Adamovich SV, et al. (2002) Haptics and virtual reality used to increase strength and improve function in chronic patients post-stroke: Two Case Reports. Neurol Phys Ther 26: 78-85.

26. Deutsch JE, Merians AS, Adamovich S, Poizner H, Burdea GC (2004) Development and application of virtual reality technology to improve hand use and gait of individuals post-stroke. Restor Neurol Neurosci 22: 371-86.

27. Holden MK (2005) Virtual environments for motor rehabilitation: Review. Cyberpsychol Behav 8: 187-211.

28. Jack D, Boian R, Merians AS, Tremaine M, Burdea GC, et al. (2001) Virtual reality-enhanced stroke rehabilitation. IEEE Transaction on Neural Syst Rehabil Eng 9: 308-318.
29. Merians AS, Jack D, Boian R, Tremaine M, Burdea GC, et al. (2002) Virtual reality augmented rehabilitation for patients following stroke. Phys Ther 82 898-915.

30. Merians AS, Poizner H, Boian R, Burdea G, Adamovich S (2006) Sensorimoto training in a virtual reality environment: Does it improve functional recovery poststroke? Neurorehabil Neural Repair 20: 252-67.

31. Murphy TH, Corbett D (2009) Plasticity during stroke recovery: From synapse to behavior. Nat Rev Neurosci 10: 861-872.

32. Langhammer B, Stanghelle JK (2003) Bobath or motor relearning programme? A follow-up one and four years post stroke. Clin Rehabil 17: 731-734.

33. Kleim JA, Jones TA (2008) Principles of experience-dependent neural plasticity: Implications for rehabilitation after brain damage. J Speech Lang Hear Res 51 : S225-S239.

34. Saposnik G, Levin M (2011) Stroke outcome research canada working group virtual reality in stroke rehabilitation: A meta- analysis and implications for clinicians. Stroke 42: 1380-1386.

35. Edmans J, Gladman J, Hilton D, Walker M, Sunderland A, et al. (2009) Clinical evaluation of a non-immersive virtual environment in stroke rehabilitation. Clin Rehabil 23: 106-16.

36. Henderson A, Korner-Bitensky N, Levin M (2007) Virtual reality in stroke rehabilitation: A systematic review of its effectiveness for upper limb motor recovery. Top Stroke Rehabil 14: 52-61.

37. Dobkin $\mathrm{BH}$ (2008) Training and exercise to drive poststroke recovery. Nat Clin Pract Neurol 4: 76-85.

38. Kleim JA, Jones TA, Schallert T (2003) Motor enrichment and the induction of plasticity before or after brain injury. Neurochem Res 28: 1757-1769.

39. Langhorne P, Coupar F, Pollock A (2009) Motor recovery after stroke: A systematic review. Lancet Neurol 8: 741-754.

40. Burdea GC (2003) Virtual rehabilitation-benefits and challenges. Methods Inf Med 42: 519-23.

41. Smith CM, Read JE, Bennie C, Hale LA, Milosavljevic S (2012) Can nonimmersive virtual reality improve physical outcomes of rehabilitation? Phys Ther Rev 17: 1-15.

42. Sanchez-Vives M, Slater M (2005) From presence to consciousness through virtual reality. Nat Rev Neurosci 6: 332-339.

43. Johansson BB (2011) Current trends in stroke rehabilitation. A review with focus on brain plasticity. Acta Neurol Scand 123: 147-159.

44. Kim JH, Jang SH, Kim CS, Jung JH, You JH (2009) Use of virtual reality to enhance balance and ambulation in chronic stroke: A double-blind, randomized controlled study. Am J of Phys Med Rehabil 88: 693-701.

45. Yavuzer G, Senel A, Atay MB, Stam HJ (2008) 'Playsationeyetoy games' improve upper extremity-related motor functioning in subacute stroke: A randomized controlled clinical trial. Eur J Phys Rehabil Med 44: 237-244.

46. Piron L, Turolla A, Agotini M, Zucconi C, Cortese F, et al. (2009) Exercises for paretic upper limb after stroke: A combined virtual-reality and telemedicine approach. J Rehabil Medi 41: 1016-20.

47. Rizzo AA, Kim GJ (2005) A SWOT analysis of the field of virtual reality rehabilitation and therapy. Teleoperators and Virtual Environments 14: 119-146.

48. Boyd LA, Winstein CJ (2001) Implicit motor-sequence learning in humans following unilateral stroke: The impact of practice and explicit knowledge. Neurosci Lett 298: 65-69.

49. Crosbie JH, Lennon S, Basford JR, McDonough SM (2007) Virtual reality in stroke rehabilitation: Still more virtual than real. Disabil Rehabil 29: 1139-1146.

50. Holden MK, Dyar TA, Dayan-Cimadoro L (2007) Telerehabilitation using a virtual environment improves upper extremity function in patients with stroke. IEEE Trans Neural Syst Rehabil Eng 15: 36-42.

51. Alankus G, Proffitt R, Kelleher C, Engsberg J (2011) Stroke therapy through motion-based games: A case study. ACM Transactions and Accessible Computing 4: 35 .

52. Wagner JM, Lang CE, Sahrmann SA, Edwards DF, Dromerick AW (2007) Sensorimotor impairments and reaching performance in subjects with poststroke hemiparesis during the first few months of recovery. Phys Ther 87 751-765. 
Citation: LeBlanc S, Paquin K, Carr K, Horton S (2013) Non-immersive Virtual Reality for Fine Motor Rehabilitation of Functional Activities in Individuals with Chronic Stroke: A Review. Aging Sci 1: 105. doi:10.4172/2329-8847.1000105

Page 8 of 8

53. Ebell MH, Siwek J, Weiss BD, et al. (2004) Strength of recommendation taxonomy (SORT): A patient-centered approach to grading evidence in the medical literature. J Am Board Fam Pract 17: 59-67.

54. World Health Organization (2002) International Classification of Functioning, Disability and Health (ICF). Switzerland.

55. Jebsen RH, Taylor N, Trieschmann RB, Trotter MJ, Howard LA (1969) An objective and standardized test of hand function. Arch Phys Med Rehabil 50: 311-319.

56. Mathiowetz V, Volland G, Kashman N, et al. (1985) Adult norms for the Box and Block Test of manual dexterity. Am J of Occup Ther 39: 386-91.

57. Mahurin RK, Debettignies BJ, Pirozzolo FJ (1991) Structured Assessment of Independent Living Skills' since it is the proper title of the assessment tool: Preliminary report of a performance measure of functional abilities in dementia. J Gerontol 46: 58-66.

58. Holden MK, Todorov E, Callahan J, Bizzi E (1999) Virtual environment training improves motor performance in two patients with stroke: case report. Neurol Rep 23: 57-67.

59. Lewis GN, Woods C, Rosie JA, Mcpherson KM (2011) Virtual reality games for rehabilitation of people with stroke: Perspectives from the users. Disabil Rehabil Assist Technol 6: 453-463.

60. Bowman T, Speier J (2006) Videoconferencing, virtual reality and homebase CIMT - Opportunities to improve access and compliance through telerehabilitation. Proceedings of the 2006 International Workshop on Virtual Rehabilitation.

61. Flynn S, Palma P, Bender A (2007) Feasibility of using the Sony PlayStation 2 gaming platform for an individual poststroke: A case report. J Neurol Phys Ther 31: $180-189$.

62. Proffitt RM, Alankus G, Kelleher CL, Engsberg JR (2011) Use of computer games as an intervention for stroke. Top Stroke Rehabil 18: 417-427. 